\title{
Smart Mobile System for Pregnancy Care using Body Sensors
}

\author{
Prasath Surendhar S., Besia Baby, Jesna S.
}

\begin{abstract}
In the recent years, child bearing has become more complex when compared to olden days. Women are at a risk of various biological changes that happens at the onset of pregnancy. In some complicated issues it's necessary to have a monitoring device that helps in proper diagnosis of the patient. With the development of Internet of Things can make all objects interconnected and it is recognized as the next technical revolution. Internet of things is used in smart homes, smart parking, smart environment, smart city and industrial areas. Internet of Things has a wide range of applications like patient data acquiring and monitoring. This can also be stored in data format where patient's parameters get transmitted through medical devices via a gateway. Thus, Internet of Things is a highly effective component in medical field because of its cheap cost and accessibility to wide range of environments. This project relies on monitoring of patient's body temperature, respiration rate, heart beat and body movement using Arduino UNO.
\end{abstract}

Key words: Technical revolution, patient's parameters, healthcare applications, Arduino UNO, respiration rate

\section{INTRODUCTION}

There has been a tremendous risk factors related to conceiving and pregnancy cases. Therefore, for normal child bearing many things has to be taken care. Not all pregnancies go to plan. Pregnancies can be a time of excitement and anticipation but some women may also experience complications such as bleeding during their pregnancy or high blood pressure or may feel dizziness which can be an indication of pre-eclamsia. Usually in pregnancy care centre there are equipments like ventilator, ECG monitoring device, sphygmomanometer, thermometer etc to measure the abnormalities of patients.

\subsection{ELECTROCARDIOGRAM}

By means of ECG leads measurements of depolarization and repolarization of cardiac muscles are monitored. This project is used to monitor the significant increase in heart rate, PR interval, deviation of $\mathrm{QRS}$ axis and $\mathrm{T}$ wave inversion.

\subsection{PULSE RATE MONITOR}

A heartbeat rate screen records the pulse progressively. During pregnancy, the measure of blood siphoned by the heart increments by 30-half. At the point when the cardiovascular yield builds, the pulse very still accelerates from an ordinary pregnancy pace of around 70 beats for every moment to 80 or 90 beats for each moment.

\subsection{HEART BEAT SENSOR}

An individual's pulse is the sound of the valves in his/her heart contracting or growing as they power blood starting with one area then onto the next. The occasions the heart pulsates every moment (BPM), is the heart beat rate and the beat of the heart that can be felt in any conduit that untruths near the skin is the beat. At the point when there is an adjustment in the worth, by methods for GSM module the in exactnesses are send by SMS to the specialist and the relatives.

\subsection{TEMPERATURE SENSOR}

A temperature sensor is used to determine the body temperature of a person. Normal body temperature of a person is 37 degree Celsius. Whereas, in case of pregnant women, it goes to 37.8 degree Celsius normally.

\section{MATERIALS AND METHOD}

We are using the Arduino development board it having atmega328p microcontroller. And we connect full 3 sensors for measuring parameter of the patient. If any things occurs to the patient it will sent the message to the respective person through the GSM wireless sensor network.

Fig a: Block Diagram for pregnancy care unit with GSM

\section{RESULT}

The mechanism of giving alert message to the patient's family and doctor is fixed. Moreover, the doctor can monitor the patient at any time. By means of application of this scalability of the proposed system in the rural areas where healthcare facilities are mostly in need, this will be highly efficient in communication with rural

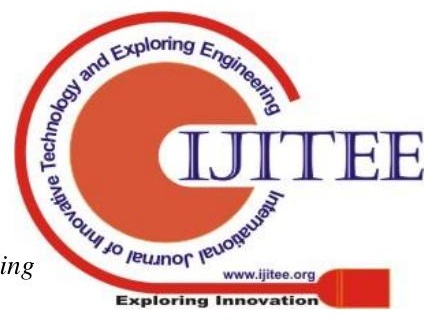




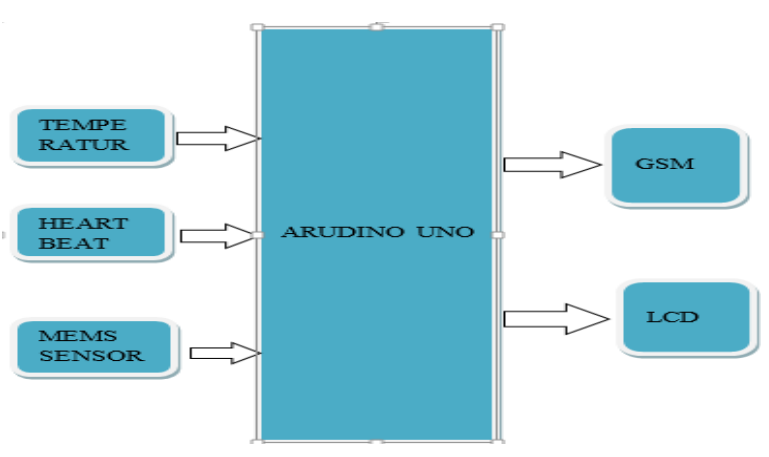

and urban patients. Moreover, this system can be used in home automation in the future. The main problems for the creation of Internet of Things for healthcare applications are monitoring all patients from various places. Thus Internet of Things in the medical field brings effective patient monitoring with low cost and also reduces the risk of managing diseases among patients. In this paper discuss about, monitoring patient's body temperature, respiration rate, heart beat and body movement using Arduino UNO. The values can be displayed in the LCD.

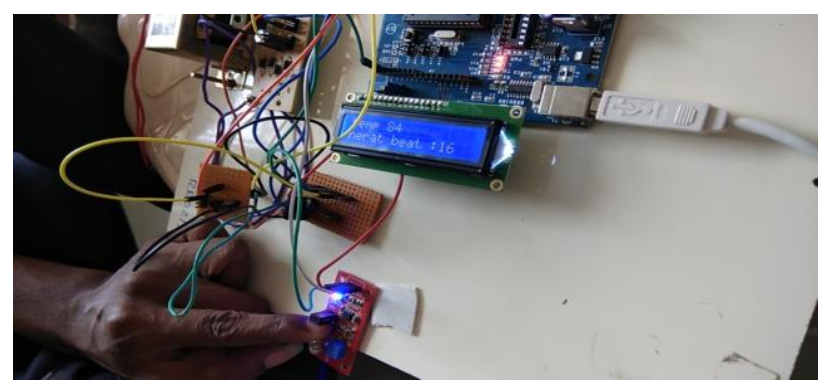

Fig b: Temperature and Heart Beat Detection
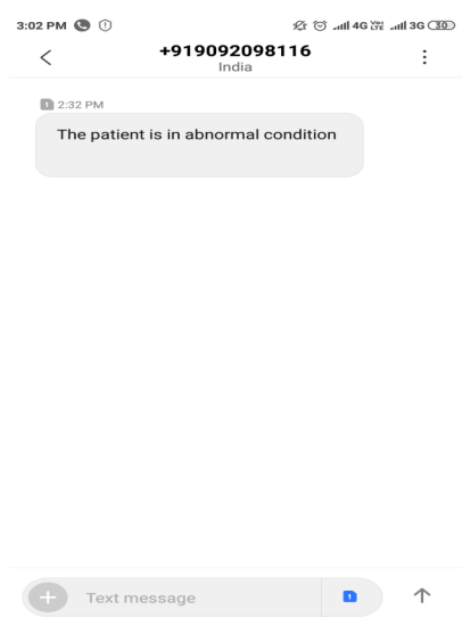

Fig c: Text Message Received By the Doctor of the Patient during Abnormal Condition

\section{CONCULSION}

By the creation of dynamic system based remote innovation with sensor-microcontroller module, the proposed NWSPMS that is in charge of deciding the wellbeing of many number of patients in the equivalent basic unit. The above proposed framework is the most reasonable framework that can be utilized for this reason. The most significant advantage of this framework is the specialists who can screen the physical and ailments of their patients from any piece of the world and thus offers directions to others paramedical staffs to take care of the patient. Along these lines the proposed framework can likewise be called as an e-emergency clinic framework, where the specialist and the patient can go to numerous patients at the same time.

\section{REFERENCES}

1. G Sanu Kumar Das, Vitthal Rathod and Akhilesh Yadav.B, "Voice Recognition Based Automation System for Medical Applications and For Physically Challenged Patients” ISCO International Conference , 2017. 2. I Ghersi, M Mariño and T Miralles, "Modern Push-Button Hospital-beds to 20th Century Mechatronic Beds", Signal processing communication power and embedded system International Conference on, 2016.

3. Mohammad Wajih Alam1, Tanin Sultana and Mohammad Sami Alam, "A Heartbeat and Temperature Measuring System for Remote Health Monitoring using Wireless Body Area Network", Medical measurements and applications IEEE International symposium, 2016.

4. Eduardo Vazquez-Santacruz, William Cruz-Santos and Mariano Gamboa-Z' u'niga, "Design and Implementation of an Intelligent System for Controlling a Robotic Hospital Bed for Patient Care Assistance", IEEE International conference on Bioinformatics and Bioengineering, 2015.

5. C. K. Das, M. W. Alam and M. I. Hoque, "A wireless heartbeat and temperature monitoring system for Remote patients." IEEE International Conference on Bioengineering, 2014.

6. Alma Secerbegovic, Aljo Muj C, Nermin Suljanovi, Midhat Nurkic, Jurij Tasic "The research mHealth platform for ECG monitoring', ConTel Conference, 2011

7. Elham Dolatabadi, Serguei Primak, Ubiquitous, "WBAN-based Electrocardiogram Monitoring System", E-Health Networking Applications Conference, 2011

8. Ramya, B.Palaniappan, Anuradha Kumari, "Embedded Patient Monitoring System', International Journal of Embedded Systems and Applications (IJESA) Vol.1, No.2, December 2011

\section{AUTHORS PROFILE}

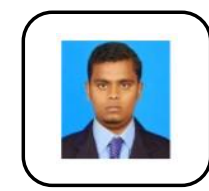

Prasath Surendhar S., was born on 1989 in Chennai. M.Tech from Department of Biomedical Engineering, VIT University in 2012. He worked as Assistant Professor - Biomedical Department -Vel Tech Multitech Engineering College - Chennai from 2013 to 2014. He currently is working Assistant Professor Biomedical Department-BIHER, Chennai

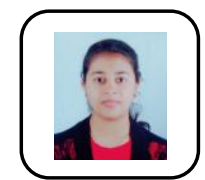

Besia Baby was born on 1996 in Kerala. She is great interest towards medical field, she opt to take graduation in (B.Tech) Biomedical Engineering from University of BIHER and doing research activities.

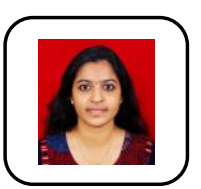

Jesna S was born on 1997 in Kerala. She is great interest towards medical field, she opt to take graduation in (B.Tech) Biomedical Engineering from University of BIHER and doing research activities. 